\title{
Tight bounds for Katona's shadow intersection theorem
}

\author{
Xizhi Liu* Dhruv Mubayi ${ }^{\dagger}$
}

May 15, 2020

\begin{abstract}
A fundamental result in extremal set theory is Katona's shadow intersection theorem, which extends the Kruskal-Katona theorem by giving a lower bound on the size of the shadow of an intersecting family of $k$-sets in terms of its size. We improve this classical result and a related result of Ahlswede, Aydinian and Khachatrian by proving tight bounds for families that can be quite small. For example, when $k=3$ our result is sharp for all families with $n$ points and at least $3 n-7$ triples.

Katona's theorem was extended by Frankl to families with matching number $s$. We improve Frankl's result by giving tight bounds for large $n$.
\end{abstract}

\section{Introduction}

Let $n \geq k \geq \ell \geq 1$. Given a family $\mathcal{H} \subset\left(\begin{array}{c}{[n]} \\ k\end{array}\right)$ the $\ell$-th shadow of $\mathcal{H}$ is

$$
\partial_{\ell} \mathcal{H}=\left\{A \in\left(\begin{array}{c}
{[n]} \\
k-\ell
\end{array}\right): \exists B \in \mathcal{H} \text { such that } A \subset B\right\}
$$

When $\ell=1$ we write $\partial \mathcal{H}$ and call $\partial \mathcal{H}$ the shadow of $\mathcal{H}$. The colex order on $\left(\begin{array}{c}{[n]} \\ k\end{array}\right)$ is defined as follows:

$$
A \prec B \text { iff } \max \{(A \backslash B) \cup(B \backslash A)\} \in B \text {. }
$$

Write $L_{m} \mathcal{H}$ to denote the set of the first $m$ elements of $\mathcal{H} \subset\left(\begin{array}{c}{[n]} \\ k\end{array}\right)$ in the colex order. When $\mathcal{H}=\left(\begin{array}{c}{[n]} \\ k\end{array}\right)$, we abuse notation by simply writing $L_{m}\left(\begin{array}{c}{[n]} \\ k\end{array}\right)$.

The celebrated Kruskal-Katona theorem states that the families in $\left(\begin{array}{c}{[n]} \\ k\end{array}\right)$ with a fixed number of sets and minimum shadow size are initial elements of the colex order.

Theorem 1.1 (Kruskal-Katona [19, 21]). For $n \geq k>\ell \geq 1$ and $\mathcal{H} \subset\left(\begin{array}{c}{[n]} \\ k\end{array}\right)$ with $|\mathcal{H}|=m$,

$$
\left|\partial_{\ell} \mathcal{H}\right| \geq\left|\partial_{\ell} L_{m}\left(\begin{array}{c}
{[n]} \\
k
\end{array}\right)\right|
$$

*Department of Mathematics, Statistics, and Computer Science, University of Illinois, Chicago, IL, 60607 USA. Email: xliu246@uic.edu. Research partially supported by NSF award DMS-1763317.

${ }^{\dagger}$ Department of Mathematics, Statistics, and Computer Science, University of Illinois, Chicago, IL, 60607 USA. Email: mubayi@uic.edu. Research partially supported by NSF award DMS-1763317. 


\subsection{Katona's shadow intersection Theorem}

The Kruskal-Katona theorem was extended to families with additional properties. One such result is due to Katona [18, about $t$-intersecting families, which are families in which every two sets have at least $t$ common elements.

Theorem 1.2 (Katona [18]). Let $n \geq k>t \geq \ell \geq 1$. If $\mathcal{H} \subset\left(\begin{array}{c}{[n]} \\ k\end{array}\right)$ is t-intersecting, then

$$
\left|\partial_{\ell} \mathcal{H}\right| \geq \frac{\left(\begin{array}{c}
2 k-t \\
k-\ell
\end{array}\right)}{\left(\begin{array}{c}
2 k-t \\
k
\end{array}\right)}|\mathcal{H}|
$$

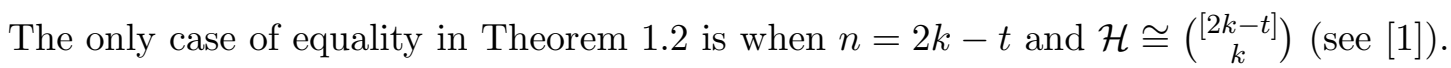

Theorem 1.2 is a foundational result in extremal set theory with many applications. Its first application was to prove a conjecture of Erdős-Ko-Rado on the maximum size of a $t$-intersecting family in $2^{[n]}$. It was used to obtain short new proofs for several classical results. For example, Frankl-Füredi [14] used it to give a short proof for the Erdős-Ko-Rado theorem, and Frankl-Tokushige [15] used it to obtain a short proof for the Hilton-Milner theorem. It also has many applications to Sperner families and other types of intersection problems [3, 4, 12, 13, 16, 22, 23, 24].

This paper is concerned with improving the bounds in Theorem 1.2 and related results about shadows of families with certain properties. In many cases the bounds we prove are best possible.

Our first result improves Theorem 1.2 for intersecting families (the case $t=1$ ) and applies to all $n>2 k$. It is convenient to define the family

$$
\operatorname{EKR}(n, k)=\left\{A \in\left(\begin{array}{c}
{[n]} \\
k
\end{array}\right): 1 \in A\right\} .
$$

Theorem 1.3. Let $n>2 k \geq 6$ and $1 \leq \ell<k$. Suppose that $\mathcal{H} \subset\left(\begin{array}{c}{[n]} \\ k\end{array}\right)$ is intersecting and

$$
|\mathcal{H}|=m>m(n, k)= \begin{cases}3 n-8, & \text { if } k=3, \\
\left(\begin{array}{l}
n-1 \\
k-1
\end{array}\right)-\left(\begin{array}{c}
n-k \\
k-1
\end{array}\right)+\left(\begin{array}{c}
n-k-2 \\
k-3
\end{array}\right)+2, & \text { if } k \geq 4 .\end{cases}
$$

Then $\left|\partial_{\ell} \mathcal{H}\right| \geq\left|\partial_{\ell} L_{m} E K R(n, k)\right|$. In particular, if for some $x \in \mathbb{R}$

$$
|\mathcal{H}|=\left(\begin{array}{l}
x-1 \\
k-1
\end{array}\right)>m(n, k)
$$

then $\left|\partial_{\ell} \mathcal{H}\right| \geq\left(\begin{array}{c}x \\ k-\ell\end{array}\right)$.

\section{Remarks.}

- For $k=3$ and $m=3 n-8$, the inequality $|\partial \mathcal{H}|<\left|\partial L_{m} E K R(n, k)\right|$ is possible (see Fact 2.16 with $t=1$ ), so Theorem 1.3 is best possible in this sense. In fact, when $k=3$ one can compute the sharp lower bound for $|\partial \mathcal{H}|$ for all intersecting families $\mathcal{H}$ using our proof method but we do not carry out all these details.

- For fixed $k>3$ and $n \rightarrow \infty$, we will lower the value of $m(n, k)$ from $(k-1+o(1))\left(\begin{array}{c}n \\ k-2\end{array}\right)$ to $(3+o(1))\left(\begin{array}{c}n \\ k-2\end{array}\right)$ in Theorem 1.10 and the constant 3 will be shown to be tight. 
Ahlswede, Aydinian, and Khachatrian [1] considered large $t$-intersecting families on $\mathbb{N}$. Let $\left(\begin{array}{l}\mathbb{N} \\ k\end{array}\right)$ denote the collection of all $k$-subsets of $\mathbb{N}$ and let

$$
\operatorname{EM}(\mathbb{N}, k, s, t)=\left\{A \in\left(\begin{array}{l}
\mathbb{N} \\
k
\end{array}\right):|A \cap[s]| \geq t\right\} .
$$

Theorem 1.4 (Ahlswede, Aydinian, and Khachatrian [1]). Let $\mathcal{H} \subset\left(\begin{array}{c}\mathbb{N} \\ k\end{array}\right)$ be at-intersecting family.

- For $1 \leq \ell \leq t<k$, there exists $m_{1}(k, t, \ell) \in \mathbb{N}$ such that if $|\mathcal{H}|=m \geq m_{1}(k, t, \ell)$, then $\left|\partial_{\ell} \mathcal{H}\right| \geq\left|\partial_{\ell} L_{m} \operatorname{EM}(\mathbb{N}, k, 2 k-2-t, k-1)\right|$.

- For $1 \leq t<\ell<k$, there exists $m_{2}(k, t, \ell) \in \mathbb{N}$ such that if $|\mathcal{H}|=m \geq m_{2}(k, t, \ell)$, then $\left|\partial_{\ell} \mathcal{H}\right| \geq\left|\partial_{\ell} L_{m} \operatorname{EM}(\mathbb{N}, k, t, t)\right|$.

For $0 \leq t \leq \min \{k, s\}$, let

$$
\operatorname{EM}(n, k, s, t)=\left\{A \subset\left(\begin{array}{c}
{[n]} \\
k
\end{array}\right):|A \cap[s]| \geq t\right\},
$$

and set $\operatorname{EM}(n, k, s, t)=\emptyset$ if $t>\min \{k, s\}$, and $\operatorname{EM}(n, k, s, t)=\left(\begin{array}{c}{[n]} \\ k\end{array}\right)$ if $n \leq s$.

For every $m \leq\left(\begin{array}{c}n-t \\ k-t\end{array}\right)$ we have $L_{m} E M(n, k, t, t)=L_{m} E M(\mathbb{N}, k, t, t)$. Therefore, Theorem 1.4 implies the following result.

Corollary 1.5. Let $1 \leq t<\ell<k$ and $\mathcal{H} \subset\left(\begin{array}{c}{[n]} \\ k\end{array}\right)$ be a t-intersecting family with $|\mathcal{H}|=$ $m>m_{2}(k, t, \ell)$. Then $\left|\partial_{\ell} \mathcal{H}\right| \geq\left|\partial_{\ell} L_{m} E M(n, k, t, t)\right|$.

However, for the case $\ell \leq t$ we show that the smallest possible size of the $\ell$-th shadow of large $t$-intersecting families on $[n]$ is different than the formula in Theorem 1.4. Let

$$
A K(n, k, t)=\left\{A \in\left(\begin{array}{c}
{[n]} \\
k
\end{array}\right):[t] \subset A \text { and }[t+1, k+1] \cap A \neq \emptyset\right\} \cup\left(\bigcup_{i \in[t]}\{[k+1] \backslash\{i\}\}\right) .
$$

Notice that $A K(n, k, t)$ and $E M(n, k, t+2, t+1)$ are both $t$-intersecting,

$$
\begin{gathered}
|A K(n, k, t)| \sim(k-t+1)\left(\begin{array}{c}
n \\
k-t-1
\end{array}\right), \\
|E M(n, k, t+2, t+1)| \sim(t+2)\left(\begin{array}{c}
n \\
k-t-1
\end{array}\right) .
\end{gathered}
$$

Our next result is a finite version of Theorem 1.4.

Theorem 1.6. Let $t \geq 1, k \geq 3,1 \leq \ell<k$, and $n>(t+1)(k-t+1)$. Suppose that $\mathcal{H} \subset\left(\begin{array}{c}{[n]} \\ k\end{array}\right)$ is t-intersecting and

$$
|\mathcal{H}|=m>m(n, k, t)= \begin{cases}\max \{|A K(n, k, t)|,|E M(n, k, t+2, t+1)|\}, & \text { if } t<\frac{k-1}{2}, \\ |E M(n, k, t+2, t+1)|, & \text { if } t \geq \frac{k-1}{2} .\end{cases}
$$

Then $\left|\partial_{\ell} \mathcal{H}\right| \geq\left|\partial_{\ell} L_{m} \operatorname{EM}(n, k, t, t)\right|$. In particular, if

$$
|\mathcal{H}|=\left(\begin{array}{l}
x-t \\
k-t
\end{array}\right)>m(n, k, t)
$$

for some $x \in \mathbb{R}$. Then $\left|\partial_{\ell} \mathcal{H}\right| \geq \sum_{i=t-\ell}^{k-\ell}\left(\begin{array}{c}t \\ i\end{array}\right)\left(\begin{array}{c}x-t \\ k-\ell-i\end{array}\right)$. For $1 \leq \ell \leq t$ the value of $m(n, k, t)$ is tight for $t \geq \frac{k-1}{2}$ and is tight up to a constant multiplicative factor independent of $n$ for $t<\frac{k-1}{2}$. 


\section{Remarks.}

- Theorem 1.6 implies that for a $t$-intersecting family $\mathcal{H} \subset\left(\begin{array}{c}{[n]} \\ k\end{array}\right)$ with large size,

$$
\frac{\left|\partial_{\ell} \mathcal{H}\right|}{|\mathcal{H}|}>\left(\begin{array}{l}
t \\
\ell
\end{array}\right) \geq \frac{\left(\begin{array}{c}
2 k-t \\
k-\ell
\end{array}\right)}{\left(\begin{array}{c}
2 k-t \\
k
\end{array}\right)}
$$

for $1 \leq \ell \leq t$ with equality in the second inequality iff $\ell=t$. Hence our bound is better than that in Theorem 1.2 (as expected since our bound is best possible).

- For $t<\frac{k-1}{2}$ we will show in the last section that the lower bound for $|\mathcal{H}|$ in Theorem 1.6 can be improved slightly.

\subsection{Frankl's theorem}

The matching number of $\mathcal{H}$, denoted by $\nu(\mathcal{H})$, is the maximum number of pairwise disjoint edges in $\mathcal{H}$. Notice that $\nu(E M(n, k, s, 1)) \leq s$ with equality iff $n \geq k s$ and

$$
|E M(n, k, s, 1)|=\left(\begin{array}{l}
n \\
k
\end{array}\right)-\left(\begin{array}{c}
n-s \\
k
\end{array}\right) \sim s\left(\begin{array}{c}
n \\
k-1
\end{array}\right) \quad(n \rightarrow \infty) .
$$

The Erdös matching conjecture [5] says that for all $n \geq(s+1) k-1$, if $\mathcal{H} \subset\left(\begin{array}{c}{[n]} \\ k\end{array}\right)$ and $\nu(\mathcal{H}) \leq s$, then

$$
|\mathcal{H}| \leq \max \left\{\left(\begin{array}{c}
(s+1) k-1 \\
k
\end{array}\right),\left(\begin{array}{l}
n \\
k
\end{array}\right)-\left(\begin{array}{c}
n-s \\
k
\end{array}\right)\right\}
$$

When $s=1$, (3) follows from the Erdős-Ko-Rado theorem [6].

Theorem 1.7 (Erdős-Ko-Rado [6]). Let $k \geq 2$ and $n \geq 2 k, \mathcal{H} \subset\left(\begin{array}{c}{[n]} \\ k\end{array}\right)$ be an intersecting family. Then $\mathcal{H} \leq\left(\begin{array}{c}n-1 \\ k-1\end{array}\right)$ and when $n>2 k$ equality holds iff $\mathcal{H} \cong E K R(n, k)$.

The Erdős matching conjecture is still open and the current record on this conjecture is due to Frankl [10].

Theorem 1.8 (Frankl [10]). Let $k \geq 2$ and $n \geq(2 s+1) k-s, \mathcal{H} \subset\left(\begin{array}{c}{[n]} \\ k\end{array}\right)$ and $\nu(\mathcal{H}) \leq s$. Then $\mathcal{H} \leq\left(\begin{array}{l}n \\ k\end{array}\right)-\left(\begin{array}{c}n-s \\ k\end{array}\right)$ with equality iff $\mathcal{H} \cong \operatorname{EM}(n, k, s, 1)$.

If we take $t=1$ in Theorem 1.2 , then every intersecting family $\mathcal{H} \subset\left(\begin{array}{c}{[n]} \\ k\end{array}\right)$ satisfies $|\partial \mathcal{H}| \geq$ $|\mathcal{H}|$. Frankl generalized this as follows.

Theorem 1.9 (Frankl [9, 10]). Let $n \geq k \geq 2$ and $\mathcal{H} \subset\left(\begin{array}{c}{[n]} \\ k\end{array}\right)$. If $\nu(\mathcal{H})=s \geq 1$, then

$$
|\partial \mathcal{H}| \geq \frac{|\mathcal{H}|}{s}
$$

with equality iff $\mathcal{H} \cong\left(\begin{array}{c}{[(s+1) k-1]} \\ k\end{array}\right)$.

Theorem 1.9 is a crucial tool in the proof of Theorem 1.8 and any improvement in Theorem 1.9 for small values of $n$ could lead to a corresponding improvement in Theorem 1.8. Our final result provides such an improvement (for large $n$ ) that is sharp if $|\mathcal{H}|$ is large. 
Theorem 1.10. For every $k \geq 3$ and every $s \geq 1$ there exists $m(n, k, s)$ such that the following holds as $n \rightarrow \infty$. Suppose that $\mathcal{H} \subset\left(\begin{array}{c}{[n]} \\ k\end{array}\right)$ satisfies $\nu(\mathcal{H}) \leq s$ and

$$
|\mathcal{H}|=m>m(n, k, s)= \begin{cases}(3+o(1))\left(\begin{array}{c}
n \\
k-2
\end{array}\right) & \text { if } s=1 \\
\left(\left(\begin{array}{c}
2 s+1 \\
2
\end{array}\right)+o(1)\right)\left(\begin{array}{c}
n \\
k-2
\end{array}\right) & \text { if } k=3 \\
\left(k\left(\begin{array}{c}
s+1 \\
2
\end{array}\right)+o(1)\right)\left(\begin{array}{c}
n \\
k-2
\end{array}\right) & \text { if } k \geq 4, s \geq 2 .\end{cases}
$$

Then

$$
|\partial \mathcal{H}| \geq\left|\partial L_{m} E M(n, k, s, 1)\right| \text {. }
$$

In particular, if $|\mathcal{H}|=\left(\begin{array}{c}x \\ k\end{array}\right)-\left(\begin{array}{c}x-s \\ k\end{array}\right)>m(n, k, s)$ for some $x \in \mathbb{R}$, then $|\partial \mathcal{H}| \geq\left(\begin{array}{c}x \\ k-1\end{array}\right)$.

The constraint $\nu(\mathcal{H}) \leq s$ above imposes the bound $|\mathcal{H}|=O\left(n^{k-1}\right)$, so the point of Theorem 1.10 is that it applies to $|\mathcal{H}| \geq(c(k, s)+o(1))\left(\begin{array}{c}n \\ k-2\end{array}\right)$ where $c(k, s)$ is obtained from (4); this is a lower order of magnitude than $n^{k-1}$. In fact, as we will show below, the order of magnitude $n^{k-2}$ is best possible for such a result and even the constant $c(k, s)$ is tight if $s=1$ or $k=3$, and is tight up to a constant factor for all other $(s, k)$.

Let $\mathcal{G}=E M(n, k, 2 s+1,2)$ and $m=|\mathcal{G}| \sim\left(\begin{array}{c}2 s+1 \\ 2\end{array}\right)\left(\begin{array}{c}n \\ k-2\end{array}\right)$ and let $x \in \mathbb{R}$ such that $\left(\begin{array}{l}x \\ k\end{array}\right)$ $\left(\begin{array}{c}x-s \\ k\end{array}\right)=m$. Since $\left(\begin{array}{c}x \\ k\end{array}\right)-\left(\begin{array}{c}x-s \\ k\end{array}\right) \sim s\left(\begin{array}{c}x \\ k-1\end{array}\right), x=\Theta\left(n^{\frac{k-2}{k-1}}\right)$. Notice that

$$
\begin{aligned}
s|\partial \mathcal{G}|-m & =s \sum_{i=1}^{k-1}\left(\begin{array}{c}
2 s+1 \\
i
\end{array}\right)\left(\begin{array}{c}
n-2 s-1 \\
k-1-i
\end{array}\right)-\sum_{i=2}^{k}\left(\begin{array}{c}
2 s+1 \\
i
\end{array}\right)\left(\begin{array}{c}
n-2 s-1 \\
k-i
\end{array}\right) \\
& =\Theta\left(n^{k-3}\right),
\end{aligned}
$$

and

$$
\begin{aligned}
s\left|\partial L_{m} E M(n, k, s, 1)\right|-m & \geq s\left(\begin{array}{c}
x \\
k-1
\end{array}\right)-\left(\left(\begin{array}{l}
x \\
k
\end{array}\right)-\left(\begin{array}{c}
x-s \\
k
\end{array}\right)\right) \\
& =\Theta\left(x^{k-2}\right)=\Theta\left(n^{\frac{(k-2)^{2}}{k-1}}\right) .
\end{aligned}
$$

Since $\frac{(k-2)^{2}}{k-1}>k-3,\left|\partial L_{m} E M(n, k, s, 1)\right|>|\partial E M(n, k, 2 s+1,2)|$ for sufficiently large $n$. Therefore, we obtain the following result.

Fact 1.11. For every $k \geq 3$ and sufficiently large $n$ there exists $\mathcal{G} \subset\left(\begin{array}{c}{[n]} \\ k\end{array}\right)$ with $\nu(\mathcal{G})=s$ and $|\mathcal{G}|=\left(1+o_{n}(1)\right)\left(\begin{array}{c}2 s+1 \\ 2\end{array}\right)\left(\begin{array}{c}n \\ k-2\end{array}\right)$ such that $|\partial \mathcal{G}|<\left|\partial L_{|\mathcal{G}|} E M(n, k, s, 1)\right|$.

It would be interesting to determine the minimum value of $c(k, s)$ such that the conclusion in Theorem 1.10 holds for all $|\mathcal{H}|>c(k, s)\left(\begin{array}{c}n \\ k-2\end{array}\right)$ and sufficiently large $n$.

\section{Proofs}

\subsection{Extension of the $k$-cascade representation}

In this section, we prove an extension of the well-known $k$-cascade representation of a number. The $k$-cascade representation plays an important role in the Kruskal-Katona theorem and the extension that we prove plays an analogous role for our theorems. As a convention, let $\left(\begin{array}{l}a \\ b\end{array}\right)=0$ if $b<0$ or $a<b$, and let $\left(\begin{array}{l}a \\ 0\end{array}\right)=1$ for all $a \geq 0$. 
For an $r$-graph $\mathcal{H}$ and a vertex set $S$ that is disjoint from $V(\mathcal{H})$ define

$$
\mathcal{H}+S=\{A \cup S: A \in \mathcal{H}\} .
$$

For every $i \in \mathbb{N}$ let $\widehat{i}=i+1$.

Lemma 2.1. Let $n \geq k \geq t \geq 0$ and $s \geq t \geq 0$. Then the following hold.

(a) $|E M(n, k, s, t)|=\left(\begin{array}{l}n \\ k\end{array}\right)-\sum_{j=0}^{t-1}\left(\begin{array}{l}s \\ j\end{array}\right)\left(\begin{array}{c}n-s \\ k-j\end{array}\right)$.

(b) For every $1 \leq m \leq|\operatorname{EM}(n, k, s, t)|$ there exist integers $a_{k}>a_{k-1}>\cdots>a_{h} \geq h \geq$ $\max \{t, 1\}$ such that

$$
L_{m} \operatorname{EM}(n, k, s, t)=E M\left(a_{k}, k, s, t\right) \cup \bigcup_{i=h}^{k-1}\left(\operatorname{EM}\left(a_{i}, i, s, t\right)+\left\{\widehat{a}_{i+1}, \ldots, \widehat{a}_{k}\right\}\right)
$$

Proof. (a) is clear. So let us consider (b).

First, it follows from the definition that the colex order of $E M\left(n^{\prime}, k, s, t\right)$ is the initial segment of the colex on $\operatorname{EM}(n, k, s, t)$ for all $n^{\prime}<n$. Let $\mathcal{F}=L_{m} E M(n, k, s, t)$. Without loss of generality we may assume that $\mathcal{F} \neq E M\left(n^{\prime}, k, s, t\right)$ for all $n^{\prime} \leq n$ since otherwise we can let $h=k$ and $a_{k}=n^{\prime}$ and we are done. So there exists $a_{k}$ such that $E M\left(a_{k}, k, s, t\right) \subset$ $\mathcal{F} \subset E M\left(a_{k}+1, k, s, t\right)$ and hence every set in $\mathcal{F} \backslash E M\left(a_{k}, k, s, t\right)$ contains $a_{k}+1$. Therefore, $\mathcal{F}=E M\left(a_{k}, k, s, t\right) \cup\left(\mathcal{F}_{k}+\left\{\widehat{a}_{k}\right\}\right)$ for some $\mathcal{F}_{k} \subset E M\left(a_{k}, k-1, s, t\right)$.

Let $m^{\prime}=\left|\mathcal{F}_{k}\right|$. Then it follows from the definition of colex order that $\mathcal{F}_{k}=L_{m^{\prime}} E M\left(a_{k}, k-\right.$ $1, s, t)$. So we can repeat the argument above to show that there exists $a_{k-1}$ such that $\mathcal{F}_{k}=E M\left(a_{k-1}, k-1, s, t\right) \cup\left(\mathcal{F}_{k-1}+\left\{\widehat{a}_{k-1}\right\}\right)$. This means that

$$
\mathcal{F}=E M\left(a_{k}, k, s, t\right) \cup\left(E M\left(a_{k-1}, k-1, s, t\right)+\left\{\widehat{a}_{k}\right\}\right) \cup\left(\mathcal{F}_{k-1}+\left\{\widehat{a}_{k}, \widehat{a}_{k-1}\right\}\right) .
$$

Inductively, one will get a decomposition of $\mathcal{F}$ as in Lemma 2.1 .

Lemma 2.2. Let $s \geq t \geq 0$ and $k \geq t$. Then, for every integers $m \geq 1$, there exists a unique representation of $m$ in the form

$$
m=\sum_{i=h}^{k}\left(\begin{array}{c}
a_{i} \\
i
\end{array}\right)-\sum_{j=0}^{t-1}\left(\begin{array}{c}
s \\
j
\end{array}\right) \sum_{i=h}^{k}\left(\begin{array}{c}
a_{i}-s \\
i-j
\end{array}\right),
$$

where $a_{k}>\cdots>a_{h} \geq h \geq \max \{t, 1\}$ are integers.

Proof. If $t=0$, then this is just the $k$-cascade representation of $m$. So we may assume that $t \geq 1$. Let $n \in \mathbb{N}$ be sufficiently large such that $m \leq|E M(n, k, s, t)|$. Then the existence of such a representation follows from Lemma 2.1 since

$$
\begin{aligned}
m=\left|L_{m} E M(n, k, s, t)\right|=\sum_{i=h}^{k}\left|E M\left(a_{i}, i, s, t\right)\right| & =\sum_{i=h}^{k}\left(\left(\begin{array}{c}
a_{i} \\
i
\end{array}\right)-\sum_{j=0}^{t-1}\left(\begin{array}{c}
s \\
j
\end{array}\right)\left(\begin{array}{c}
a_{i}-s \\
i-j
\end{array}\right)\right) \\
& =\sum_{i=h}^{k}\left(\begin{array}{c}
a_{i} \\
i
\end{array}\right)-\sum_{j=0}^{t-1}\left(\begin{array}{c}
s \\
j
\end{array}\right) \sum_{i=h}^{k}\left(\begin{array}{c}
a_{i}-s \\
i-j
\end{array}\right) .
\end{aligned}
$$


Next, we prove the uniqueness of such representation of $m$. Suppose that there exists $a_{k}>\cdots>a_{h} \geq h \geq t$ and $b_{k}>\cdots>b_{h^{\prime}} \geq h^{\prime} \geq t$ such that

$$
\sum_{i=h}^{k}\left(\begin{array}{c}
a_{i} \\
i
\end{array}\right)-\sum_{j=0}^{t-1}\left(\begin{array}{c}
s \\
j
\end{array}\right) \sum_{i=h}^{k}\left(\begin{array}{c}
a_{i}-s \\
i-j
\end{array}\right)=m=\sum_{i=h^{\prime}}^{k}\left(\begin{array}{c}
b_{i} \\
i
\end{array}\right)-\sum_{j=0}^{t-1}\left(\begin{array}{c}
s \\
j
\end{array}\right) \sum_{i=h^{\prime}}^{k}\left(\begin{array}{c}
b_{i}-s \\
i-j
\end{array}\right) .
$$

Without loss of generality we may assume that $a_{k} \neq b_{k}$ since otherwise we can consider $m^{\prime}=m-\left(\left(\begin{array}{c}a_{k} \\ i\end{array}\right)-\sum_{j=0}^{t-1}\left(\begin{array}{c}s \\ j\end{array}\right)\left(\begin{array}{c}a_{k}-s \\ i-j\end{array}\right)\right)$ instead. Let

$$
\mathcal{F}_{a}=E M\left(a_{k}, k, s, t\right) \cup \bigcup_{i=h}^{k-1}\left(E M\left(a_{i}, i, s, t\right)+\left\{\widehat{a}_{i+1}, \ldots, \widehat{a}_{k}\right\}\right)
$$

and

$$
\mathcal{F}_{b}=E M\left(b_{k}, k, s, t\right) \cup \bigcup_{i=h^{\prime}}^{k-1}\left(E M\left(b_{i}, i, s, t\right)+\left\{\widehat{b}_{i+1}, \ldots, \widehat{b}_{k}\right\}\right)
$$

Then

$$
\left|\mathcal{F}_{a}\right|=\sum_{i=h}^{k}\left(\begin{array}{c}
a_{i} \\
i
\end{array}\right)-\sum_{j=0}^{t-1}\left(\begin{array}{c}
s \\
j
\end{array}\right) \sum_{i=h}^{k}\left(\begin{array}{c}
a_{i}-s \\
i-j
\end{array}\right)
$$

and

$$
\left|\mathcal{F}_{b}\right|=\sum_{i=h^{\prime}}^{k}\left(\begin{array}{c}
b_{i} \\
i
\end{array}\right)-\sum_{j=0}^{t-1}\left(\begin{array}{c}
s \\
j
\end{array}\right) \sum_{i=h^{\prime}}^{k}\left(\begin{array}{c}
b_{i}-s \\
i-j
\end{array}\right) .
$$

Without loss of generality we may assume that $a_{k} \geq b_{k}+1$. However, notice that in this case $\mathcal{F}_{b}$ is a proper subset of $\mathcal{F}_{a}$, since every set of $\mathcal{F}_{b}$ has maximum element at most $b_{k}+1 \leq a_{k}$. This contradicts (5).

\subsection{Shifting}

For every $A \in \mathcal{H}$ and $1 \leq i<j \leq n$ define

$$
S_{i j}(A)= \begin{cases}(A \backslash\{j\}) \cup\{i\}, & \text { if } j \in A, i \notin A, \text { and }(A \backslash\{j\}) \cup\{i\} \notin \mathcal{H}, \\ A, & \text { otherwise. }\end{cases}
$$

Let $S_{i j}(\mathcal{H})=\left\{S_{i j}(A): A \in \mathcal{H}\right\}$ and call $\mathcal{H}$ shifted if $\mathcal{H}=S_{i j}(\mathcal{H})$ for all $1 \leq i<j \leq n$.

Fact 2.3 (see [8] ). The following statements hold for all $\mathcal{H} \subset\left(\begin{array}{c}{[n]} \\ k\end{array}\right)$ and all $1 \leq i<j \leq n$ and all $1 \leq t, \ell \leq k-1$.

- $|\mathcal{H}|=\left|S_{i j}(\mathcal{H})\right|$.

- $\partial_{\ell} S_{i j}(\mathcal{H}) \subset S_{i j}\left(\partial_{\ell} \mathcal{H}\right)$ and in particular, $\left|S_{i j}\left(\partial_{\ell} \mathcal{H}\right)\right| \geq\left|\partial_{\ell} S_{i j}(\mathcal{H})\right|$

- $\nu\left(S_{i j}(\mathcal{H})\right) \leq \nu(\mathcal{H})$.

- If $\mathcal{H}$ is $t$-intersecting, then $S_{i j}(\mathcal{H})$ is also t-intersecting. 


\subsection{Main Lemma}

Fact 2.3 shows that it suffices to consider shifted families in all proofs in this paper. The main technical statement in this work is Lemma 2.5 below which is a generalization of the Kruskal-Katona theorem. For two families $\mathcal{H}_{1}$ and $\mathcal{H}_{2}$ we write $\mathcal{H}_{1} \subset \mathcal{H}_{2}$ if $\mathcal{H}_{1}$ is isomorphic to a subgraph of $\mathcal{H}_{2}$.

Given a family $\mathcal{H}$, let $\mathcal{H}(1)=\{A \backslash\{1\}: 1 \in A \in \mathcal{H}\}$ and $\mathcal{H}(\overline{1})=\{A \in \mathcal{H}: 1 \notin A\}$. It is easy to see that if $\mathcal{H}$ is shifted, then $\partial \mathcal{H}(\overline{1}) \subset \mathcal{H}(1)$ and hence $|\partial \mathcal{H}|=|\mathcal{H}(1)|+|\partial \mathcal{H}(1)|$.

Lemma 2.4. Let $n \geq k \geq t \geq 0$ and $s \geq t \geq 0$. Suppose that

$$
m=\sum_{i=h}^{k}\left(\begin{array}{c}
a_{i} \\
i
\end{array}\right)-\sum_{j=0}^{t-1}\left(\begin{array}{l}
s \\
j
\end{array}\right) \sum_{i=h}^{k}\left(\begin{array}{c}
a_{i}-s \\
i-j
\end{array}\right)
$$

for integers $a_{k}>\cdots>a_{h} \geq \max \{t, 1\}$. Then for $1 \leq \ell \leq k-1$

$$
\left|\partial_{\ell} L_{m} E M(n, k, s, t)\right|=\sum_{i=h}^{k}\left(\begin{array}{c}
a_{i} \\
i-\ell
\end{array}\right)-\sum_{j=0}^{t-1-\ell}\left(\begin{array}{l}
s \\
j
\end{array}\right) \sum_{i=h}^{k}\left(\begin{array}{c}
a_{i}-s \\
i-\ell-j
\end{array}\right) .
$$

Proof. Fix $1 \leq \ell \leq k-1$. By Lemma 2.1 ,

$$
L_{m} \operatorname{EM}(n, k, s, t)=E M\left(a_{k}, k, s, t\right) \cup \bigcup_{i=h}^{k-1}\left(E M\left(a_{i}, i, s, t\right)+\left\{\widehat{a}_{i+1}, \ldots, \widehat{a}_{k}\right\}\right) .
$$

Notice that

$$
\partial \operatorname{EM}\left(a_{k}, k, s, t\right)=\operatorname{EM}\left(a_{k}, k-1, s, t-1\right),
$$

and for every $h \leq i \leq k-1$ we have

$$
\begin{array}{r}
\partial\left(E M\left(a_{i}, i, s, t\right)+\left\{\widehat{a}_{i+1}, \ldots, \widehat{a}_{k}\right\}\right)=\left(E M\left(a_{i}, i-1, s, t-1\right)+\left\{\widehat{a}_{i+1}, \ldots, \widehat{a}_{k}\right\}\right) \cup \\
\bigcup_{j=i+1}^{k}\left(E M\left(a_{i}, i, s, t\right)+\left\{\widehat{a}_{i+1}, \ldots, \widehat{a}_{k}\right\} \backslash\left\{\widehat{a}_{j}\right\}\right) .
\end{array}
$$

On the other hand, for all $h \leq i<j \leq k-2$ since $a_{j}>a_{i}$,

$$
E M\left(a_{i}, i, s, t\right)+\left\{\widehat{a}_{i+1}, \ldots, \widehat{a}_{k}\right\} \backslash\left\{\widehat{a}_{j}\right\} \subset E M\left(a_{j}, j-1, s, t-1\right)+\left\{\widehat{a}_{j+1}, \ldots, \widehat{a}_{k}\right\} .
$$

For all $h \leq i \leq k-1$ since $a_{k}>a_{i}$,

$$
E M\left(a_{i}, i, s, t\right)+\left\{\widehat{a}_{i+1}, \ldots, \widehat{a}_{k}\right\} \backslash\left\{\widehat{a}_{k}\right\} \subset E M\left(a_{k}, k-1, s, t-1\right) .
$$

Therefore,

$$
\partial L_{m} \operatorname{EM}(n, k, s, t)=\bigcup_{i=h}^{k}\left(E M\left(a_{i}, i-1, s, t-1\right)+\left\{\widehat{a}_{i+1}, \ldots, \widehat{a}_{k}\right\}\right),
$$

and inductively we obtain for all $1 \leq \ell \leq k-1$

$$
\partial_{\ell} L_{m} E M(n, k, s, t)=\bigcup_{i=h}^{k}\left(E M\left(a_{i}, i-\ell, s, t-\ell\right)+\left\{\widehat{a}_{i+1}, \ldots, \widehat{a}_{k}\right\}\right) .
$$


Therefore,

$$
\begin{aligned}
\left|\partial_{\ell} L_{m} E M(n, k, s, t)\right| & =\sum_{i=h}^{k}\left|E M\left(a_{i}, i-\ell, s, t-\ell\right)\right| \\
& =\sum_{i=h}^{k}\left(\left(\begin{array}{c}
a_{i} \\
i-\ell
\end{array}\right)-\sum_{j=0}^{t-1-\ell}\left(\begin{array}{l}
s \\
j
\end{array}\right)\left(\begin{array}{c}
a_{i} \\
i-\ell-j
\end{array}\right)\right) \\
& =\sum_{i=h}^{k}\left(\begin{array}{c}
a_{i} \\
i-\ell
\end{array}\right)-\sum_{j=0}^{t-1-\ell}\left(\begin{array}{l}
s \\
j
\end{array}\right) \sum_{i=h}^{k}\left(\begin{array}{c}
a_{i}-s \\
i-\ell-j
\end{array}\right) .
\end{aligned}
$$

Lemma 2.5. Let $s \geq t \geq 0$. If $\mathcal{H} \subset E M(n, k, s, t)$ and $|\mathcal{H}|=m$, then

$$
|\partial \mathcal{H}| \geq\left|\partial L_{m} E M(n, k, s, t)\right| .
$$

Proof. By Lemma 2.2, there exists $a_{k}>\cdots>a_{h} \geq \max \{t, 1\}$ such that

$$
m=\sum_{i=h}^{k}\left(\begin{array}{c}
a_{i} \\
i
\end{array}\right)-\sum_{j=0}^{t-1}\left(\begin{array}{l}
s \\
j
\end{array}\right) \sum_{i=h}^{k}\left(\begin{array}{c}
a_{i}-s \\
i-j
\end{array}\right) .
$$

Then, by Lemma 2.4 it suffices to show that

$$
|\partial \mathcal{H}| \geq \sum_{i=h}^{k}\left(\begin{array}{c}
a_{i} \\
i-1
\end{array}\right)-\sum_{j=0}^{t-2}\left(\begin{array}{l}
s \\
j
\end{array}\right) \sum_{i=h}^{k}\left(\begin{array}{c}
a_{i}-s \\
i-1-j
\end{array}\right) .
$$

We prove this statement by induction on $k, s, t$. When $s=0$ or $k=1$ the statement is trivially true. When $t=0$ the statement follows from the Kruskal-Katona theorem. So we may assume that $s \geq t \geq 1$ and $k \geq 2$.

Claim 2.6. $|\mathcal{H}(1)| \geq \sum_{i=h}^{k}\left(\begin{array}{c}a_{i}-1 \\ i-1\end{array}\right)-\sum_{j=0}^{t-2}\left(\begin{array}{c}s-1 \\ j\end{array}\right) \sum_{i=h}^{k}\left(\begin{array}{c}a_{i}-s \\ i-1-j\end{array}\right)$.

Proof of Claim [2.6. Suppose not. Then

$$
\begin{aligned}
|\mathcal{H}(\overline{1})| & =|\mathcal{H}|-|\mathcal{H}(1)| \\
& >\sum_{i=h}^{k}\left(\begin{array}{c}
a_{i} \\
i
\end{array}\right)-\sum_{j=0}^{t-1}\left(\begin{array}{c}
s \\
j
\end{array}\right) \sum_{i=h}^{k}\left(\begin{array}{c}
a_{i}-s \\
i-j
\end{array}\right)-\left(\sum_{i=h}^{k}\left(\begin{array}{c}
a_{i}-1 \\
i-1
\end{array}\right)-\sum_{j=0}^{t-2}\left(\begin{array}{c}
s-1 \\
j
\end{array}\right) \sum_{i=h}^{k}\left(\begin{array}{c}
a_{i}-s \\
i-1-j
\end{array}\right)\right) \\
& =\sum_{i=h}^{k}\left(\begin{array}{c}
a_{i} \\
i
\end{array}\right)-\sum_{i=h}^{k}\left(\begin{array}{c}
a_{i}-1 \\
i-1
\end{array}\right)-\sum_{j=0}^{t-1}\left(\left(\begin{array}{c}
s \\
j
\end{array}\right)-\left(\begin{array}{c}
s-1 \\
j-1
\end{array}\right)\right) \sum_{i=h}^{k}\left(\begin{array}{c}
a_{i}-s \\
i-j
\end{array}\right) \\
& =\sum_{i=h}^{k}\left(\begin{array}{c}
a_{i}-1 \\
i
\end{array}\right)-\sum_{j=0}^{t-1}\left(\begin{array}{c}
s-1 \\
j
\end{array}\right) \sum_{i=h}^{k}\left(\begin{array}{c}
a_{i}-s \\
i-j
\end{array}\right) .
\end{aligned}
$$

Since $\mathcal{H}(\overline{1}) \subset E M(n, k, s-1, t)$, by the induction hypothesis

$$
|\partial \mathcal{H}(\overline{1})|>\sum_{i=h}^{k}\left(\begin{array}{c}
a_{i}-1 \\
i-1
\end{array}\right)-\sum_{j=0}^{t-2}\left(\begin{array}{c}
s-1 \\
j
\end{array}\right) \sum_{i=h}^{k}\left(\begin{array}{c}
a_{i}-s \\
i-1-j
\end{array}\right)>|\mathcal{H}(1)|,
$$

which contradicts the assumption that $\mathcal{H}$ is shifted. 
Since $\mathcal{H}(1) \subset E M(n, k-1, s-1, t-1)$, by the induction hypothesis and Claim 2.6,

$$
\begin{aligned}
|\partial \mathcal{H}| \geq & |\mathcal{H}(1)|+|\partial \mathcal{H}(1)| \\
\geq & \sum_{i=h}^{k}\left(\begin{array}{c}
a_{i}-1 \\
i-1
\end{array}\right)-\sum_{j=0}^{t-2}\left(\begin{array}{c}
s-1 \\
j
\end{array}\right) \sum_{i=h}^{k}\left(\begin{array}{c}
a_{i}-s \\
i-1-j
\end{array}\right) \\
& +\sum_{i=h}^{k}\left(\begin{array}{c}
a_{i}-1 \\
i-2
\end{array}\right)-\sum_{j=0}^{t-3}\left(\begin{array}{c}
s-1 \\
j
\end{array}\right) \sum_{i=h}^{k}\left(\begin{array}{c}
a_{i}-s \\
i-2-j
\end{array}\right) \\
= & \sum_{i=h}^{k}\left(\begin{array}{c}
a_{i} \\
i-1
\end{array}\right)-\sum_{j=0}^{t-2}\left(\left(\begin{array}{c}
s-1 \\
j
\end{array}\right)+\left(\begin{array}{c}
s-1 \\
j-1
\end{array}\right)\right) \sum_{i=h}^{k}\left(\begin{array}{c}
a_{i}-s \\
i-1-j
\end{array}\right) \\
= & \sum_{i=h}^{k}\left(\begin{array}{c}
a_{i} \\
i-1
\end{array}\right)-\sum_{j=0}^{t-2}\left(\begin{array}{c}
s \\
j
\end{array}\right) \sum_{i=h}^{k}\left(\begin{array}{c}
a_{i}-s \\
i-1-j
\end{array}\right) .
\end{aligned}
$$

This completes the proof of Lemma 2.5 .

Corollary 2.7. Let $s \geq t \geq 0$ and $1 \leq \ell \leq k-1$. Suppose that $\mathcal{H} \subset E M(n, k, s, t)$ and $|\mathcal{H}|=m$. Then $\left|\partial_{\ell} \mathcal{H}\right| \geq\left|\partial_{\ell} L_{m} E M(n, k, s, t)\right|$.

Proof. Similar to the proof of Lemma 2.5, it suffices to show that if for some integers $a_{k}>\cdots>a_{h} \geq h \geq \max \{t, 1\}$

$$
|\mathcal{H}|=\sum_{i=h}^{k}\left(\begin{array}{c}
a_{i} \\
i
\end{array}\right)-\sum_{j=0}^{t-1}\left(\begin{array}{c}
s \\
j
\end{array}\right) \sum_{i=h}^{k}\left(\begin{array}{c}
a_{i}-s \\
i-j
\end{array}\right)
$$

then

$$
\left|\partial_{\ell} \mathcal{H}\right| \geq \sum_{i=h}^{k}\left(\begin{array}{c}
a_{i} \\
i-\ell
\end{array}\right)-\sum_{j=0}^{t-1-\ell}\left(\begin{array}{l}
s \\
j
\end{array}\right) \sum_{i=h}^{k}\left(\begin{array}{c}
a_{i}-s \\
i-\ell-j
\end{array}\right) .
$$

We proceed by induction on $\ell$. When $\ell=1$, this is Lemma 2.5. So we may assume that $\ell \geq 2$. By the induction hypothesis

$$
\left|\partial_{\ell-1} \mathcal{H}\right| \geq \sum_{i=h}^{k}\left(\begin{array}{c}
a_{i} \\
i-\ell+1
\end{array}\right)-\sum_{j=0}^{t-\ell}\left(\begin{array}{l}
s \\
j
\end{array}\right) \sum_{i=h}^{k}\left(\begin{array}{c}
a_{i}-s \\
i-\ell+1-j
\end{array}\right) .
$$

Since $\partial_{\ell-1} \mathcal{H} \subset E M(n, k, s, t-\ell+1)$, by Lemma 2.5 ,

$$
\left|\partial_{\ell} \mathcal{H}\right|=\left|\partial \partial_{\ell-1} \mathcal{H}\right| \geq \sum_{i=h}^{k}\left(\begin{array}{c}
a_{i} \\
i-\ell
\end{array}\right)-\sum_{j=0}^{t-\ell-1}\left(\begin{array}{l}
s \\
j
\end{array}\right) \sum_{i=h}^{k}\left(\begin{array}{c}
a_{i}-s \\
i-\ell-j
\end{array}\right) .
$$

This completes the proof of Corollary 2.7,

The same induction argument as above gives the following technically simpler version of Corollary 2.7.

Lemma 2.8 (Simplified version of Lemma 2.5). Let $s \geq t \geq 0$ and $1 \leq \ell \leq k-1$. Suppose that $\mathcal{H} \subset \operatorname{EM}(n, k, s, t)$ and $|\mathcal{H}|=\left(\begin{array}{l}x \\ k\end{array}\right)-\sum_{j=0}^{t-1}\left(\begin{array}{c}s \\ j\end{array}\right)\left(\begin{array}{c}x-s \\ k-j\end{array}\right)$ for some $x \in \mathbb{R}$. Then $\left|\partial_{\ell} \mathcal{H}\right| \geq\left(\begin{array}{c}x \\ k-\ell\end{array}\right)-\sum_{j=0}^{t-1-\ell}\left(\begin{array}{c}s \\ j\end{array}\right)\left(\begin{array}{c}x-s \\ k-\ell-j\end{array}\right)$. 
Let

$$
\begin{aligned}
H M(n, k, s, t)= & \left\{A \in\left(\begin{array}{c}
{[n]} \\
k
\end{array}\right):|A \cap[s-1]| \geq 1\right\} \cup \\
& \left\{A \in\left(\begin{array}{c}
{[n]} \\
k
\end{array}\right): s \in A \text { and }|A \cap[s+1, s+t]| \geq 1\right\} .
\end{aligned}
$$

Note that there is no constraint on the relation between $s$ and $t$ for $H M(n, k, s, t)$.

Similar to Lemmas 2.1, 2.2, and 2.4 we have the following result for $H M(n, k, s, t)$.

Lemma 2.9. Let $n \geq k$. Then the following hold.

(a) $|H M(n, k, s, t)|=\left(\begin{array}{c}n \\ k\end{array}\right)-\left(\begin{array}{c}n-s \\ k\end{array}\right)-\left(\begin{array}{c}n-s-t \\ k-1\end{array}\right)$ and $|\partial H M(n, k, s, t)|=\left(\begin{array}{c}n \\ k-1\end{array}\right)$.

(b) For every $m \leq|H M(n, k, s, t)|$ there exist integers $a_{k}>\cdots>a_{h} \geq h \geq 1$ such that

$$
L_{m} H M(n, k, s, t)=H M\left(a_{k}, k, s, t\right) \cup \bigcup_{i=h}^{k-1}\left(H M\left(a_{i}, i, s, t\right)+\left\{\widehat{a}_{i+1}, \ldots, \widehat{a}_{k}\right\}\right) .
$$

(c) For every $m \geq 1$ there exists a unique sequence of integers $a_{k}>\cdots>a_{h} \geq h \geq 1$ such that

$$
m=\sum_{i=h}^{k}\left(\begin{array}{c}
a_{i} \\
i
\end{array}\right)-\sum_{i=h}^{k}\left(\begin{array}{c}
a_{i}-s \\
i
\end{array}\right)-\sum_{i=h}^{k}\left(\begin{array}{c}
a_{i}-s-t \\
i-1
\end{array}\right) .
$$

(d) If $m$ is given by the equation above, then

$$
\left|\partial L_{m} H M(n, k, s, t)\right|=\sum_{i=h}^{k}\left(\begin{array}{c}
a_{i} \\
i-1
\end{array}\right) .
$$

Lemma 2.10. If $\mathcal{H} \subset H M(n, k, s, t)$ and $|\mathcal{H}|=m$, then $|\partial \mathcal{H}| \geq\left|\partial L_{m} H M(n, k, s, t)\right|$. In particular, if $|\mathcal{H}|=\sum_{i=h}^{k}\left(\begin{array}{c}a_{i} \\ i\end{array}\right)-\sum_{i=h}^{k}\left(\begin{array}{c}a_{i}-s \\ i\end{array}\right)-\sum_{i=h}^{k}\left(\begin{array}{c}a_{i}-s-t \\ i-1\end{array}\right)$ for some integers $a_{k}>\cdots>$ $a_{h} \geq h \geq 1$, then $|\partial \mathcal{H}| \geq \sum_{i=h}^{k}\left(\begin{array}{c}a_{i} \\ i-1\end{array}\right)$.

Proof. Let $a_{k}>\cdots>a_{h} \geq h \geq 1$ be integers such that

$$
m=\sum_{i=h}^{k}\left(\begin{array}{c}
a_{i} \\
i
\end{array}\right)-\sum_{i=h}^{k}\left(\begin{array}{c}
a_{i}-s \\
i
\end{array}\right)-\sum_{i=h}^{k}\left(\begin{array}{c}
a_{i}-s-t \\
i-1
\end{array}\right) .
$$

Then by Lemma 2.9, it suffices to show

$$
|\partial \mathcal{H}| \geq \sum_{i=h}^{k}\left(\begin{array}{c}
a_{i} \\
i-1
\end{array}\right)=\left|\partial L_{m} H M(n, k, s, t)\right| .
$$

We proceed by induction on $s$ and $t$. When $s=0$, this is trivially true. When $t=0$, we have $H M(n, k, s, 0)=E M(n, k, s, 1)$, so the conclusion follows from Lemma 2.5. So we may assume that $s \geq 1$ and $t \geq 1$.

Claim 2.11. $|\mathcal{H}(1)| \geq \sum_{i=h}^{k}\left(\begin{array}{c}a_{i}-1 \\ i-1\end{array}\right)$ 
Proof of Claim 2.11. Suppose not. Then

$$
|\mathcal{H}(\overline{1})|=|\mathcal{H}|-|\mathcal{H}(1)|>\sum_{i=h}^{k}\left(\begin{array}{c}
a_{i}-1 \\
i
\end{array}\right)-\sum_{i=h}^{k}\left(\begin{array}{c}
a_{i}-s \\
i
\end{array}\right)-\sum_{i=h}^{k}\left(\begin{array}{c}
a_{i}-s-t \\
i-1
\end{array}\right) .
$$

Since $\mathcal{H}(\overline{1}) \subset H M(n, k, s-1, t)$, by the induction hypothesis $|\partial \mathcal{H}(\overline{1})|>\sum_{i=h}^{k}\left(\begin{array}{c}a_{i}-1 \\ i-1\end{array}\right)>$ $|\mathcal{H}(1)|$, which contradicts the assumption that $\mathcal{H}$ is shifted.

Now, by Claim 2.11 and the Kruskal-Katona theorem,

$$
|\partial \mathcal{H}| \geq|\mathcal{H}(1)|+|\partial \mathcal{H}(1)| \geq \sum_{i=h}^{k}\left(\begin{array}{c}
a_{i}-1 \\
i-1
\end{array}\right)+\sum_{i=h}^{k}\left(\begin{array}{c}
a_{i}-1 \\
i-2
\end{array}\right)=\sum_{i=h}^{k}\left(\begin{array}{c}
a_{i} \\
i-1
\end{array}\right) .
$$

This completes the proof of Lemma 2.10.

Similarly, the same induction argument as above gives the following technically simpler version of Lemma 2.10,

Lemma 2.12 (Simplified version of Lemma 2.10). Suppose that $\mathcal{H} \subset H M(n, k, s, t)$ and $|\mathcal{H}|=\left(\begin{array}{l}x \\ k\end{array}\right)-\left(\begin{array}{c}x-s \\ k\end{array}\right)-\left(\begin{array}{c}x-s-t \\ k-1\end{array}\right)$ for some $x \in \mathbb{R}$. Then $|\partial \mathcal{H}| \geq\left(\begin{array}{c}x \\ k-1\end{array}\right)$.

\subsection{Proof of Theorem 1.3}

The proof of Theorem 1.3 uses the following structural theorem for intersecting families.

For $1 \leq t \leq k-1$ let

$$
\begin{aligned}
H M(n, k, t)= & \{A \in E K R(n, k): A \cap[2, k] \neq \emptyset \text { or }[k+1, k+t] \subset A\} \cup \\
& \bigcup_{i=1}^{t}\{\{2, \ldots, k, k+i\}\} .
\end{aligned}
$$

Note that $|H M(n, k, 2)|=\left(\begin{array}{c}n-1 \\ k-1\end{array}\right)-\left(\begin{array}{c}n-k \\ k-1\end{array}\right)+\left(\begin{array}{c}n-k-2 \\ k-3\end{array}\right)+2$ and $H M(n, k, 1)$ is the extremal configuration in the Hilton-Milner theorem on nontrivial intersecting families.

Theorem 2.13 (Han and Kohayakawa, [17]). Let $k \geq 3$ and $n>2 k$ and let $\mathcal{H}$ be an $n$-vertex intersecting k-graph. If $\mathcal{H} \not \subset E K R(n, k)$ and $\mathcal{H} \not \subset H M(n, k, 1)$ and for $k=3$ $\mathcal{H} \not \subset \operatorname{EM}(n, 3,3,2)$ as well, then $|\mathcal{H}| \leq|H M(n, k, 2)|$.

Proof of Theorem 1.3. By the assumption on the size of $\mathcal{H}$ and Theorem 2.13, for $k \geq 4$ either $\mathcal{H} \subset E K R(n, k)$ or $\mathcal{H} \subset H M(n, k, 1)$, and for $k=3$ we have $\mathcal{H} \subset E K R(n, 3)$.

Suppose that $k=3$. Since $\mathcal{H} \subset E K R(n, 3)=E M(n, 3,1,1)$, by Corollary 2.7, $\left|\partial_{\ell} \mathcal{H}\right| \geq$ $\left|\partial_{\ell} L_{m} E K R(n, 3)\right|$ for $1 \leq \ell \leq 2$.

Now suppose that $k \geq 4$. Let $a_{k}>\cdots>a_{h} \geq h \geq 1$ be integers such that $|\mathcal{H}|=$ $\sum_{i=h}^{k}\left(\begin{array}{c}a_{i} \\ i\end{array}\right)-\sum_{i=h}^{k}\left(\begin{array}{c}a_{i}-1 \\ i\end{array}\right)$. If $\mathcal{H} \subset \operatorname{EKR}(n, k)=\operatorname{EM}(n, k, 1,1)$, then by Corollary 2.7. $\left|\partial_{\ell} \mathcal{H}\right| \geq\left|\partial_{\ell} L_{m} \operatorname{EM}(n, k, 1,1)\right|=\left|\partial_{\ell} L_{m} E K R(n, k)\right|$ and we are done. So we may assume that $\mathcal{H} \subset H M(n, k, 1)$ and we are going to show that $\left|\partial_{\ell} \mathcal{H}\right|>\sum_{i=h}^{k}\left(\begin{array}{c}a_{i} \\ i-\ell\end{array}\right)=$ $\left|\partial_{\ell} L_{m} E K R(n, k)\right|$ in this case.

Suppose that $\left|\partial_{\ell} \mathcal{H}\right| \leq \sum_{i=h}^{k}\left(\begin{array}{c}a_{i} \\ i-\ell\end{array}\right)$. Let $\mathcal{H}^{\prime}=\mathcal{H} \backslash\{\{2, \ldots, k+1\}\}$ and note that $\left|\partial_{\ell} \mathcal{H}^{\prime}\right| \leq$ $\left|\partial_{\ell} \mathcal{H}\right| \leq \sum_{i=h}^{k}\left(\begin{array}{c}a_{i} \\ i-\ell\end{array}\right)$. Applying the the contrapositive of the Kruskal-Katona theorem to 
$\mathcal{H}^{\prime}$ we obtain $\left|\partial \mathcal{H}^{\prime}\right| \leq \sum_{i=h}^{k}\left(\begin{array}{c}a_{i} \\ i-1\end{array}\right)$. On the other hand, since $\mathcal{H} \subset H M(n, k, 1), \mathcal{H}^{\prime} \subset$ $H M(n, k, 1) \backslash\{\{2, \ldots, k+1\}\}=H M(n, k, 1, k)$. So applying the contrapositive of Lemma 2.10 to $\mathcal{H}^{\prime}$ we obtain

$$
\begin{aligned}
|\mathcal{H}| \leq\left|\mathcal{H}^{\prime}\right|+1 & \leq \sum_{i=h}^{k}\left(\begin{array}{c}
a_{i} \\
i
\end{array}\right)-\sum_{i=h}^{k}\left(\begin{array}{c}
a_{i}-1 \\
i
\end{array}\right)-\sum_{i=h}^{k}\left(\begin{array}{c}
a_{i}-1-k \\
i-1
\end{array}\right)+1 \\
& =\sum_{i=h}^{k}\left(\begin{array}{c}
a_{i}-1 \\
i-1
\end{array}\right)-\sum_{i=h}^{k}\left(\begin{array}{c}
a_{i}-1-k \\
i-1
\end{array}\right)+1 .
\end{aligned}
$$

Claim 2.14. $a_{k} \geq 2 k$ and if $a_{k}=2 k$ then $a_{k-1}=2 k-1$.

Proof of Claim 2.14. First, suppose that $a_{k} \leq 2 k-1$. Then

$$
\begin{aligned}
|\mathcal{H}| & \leq \sum_{i=h}^{k}\left(\begin{array}{c}
a_{i}-1 \\
i-1
\end{array}\right)-\sum_{i=h}^{k}\left(\begin{array}{c}
a_{i}-1-k \\
i-1
\end{array}\right)+1 \\
& \leq \sum_{i=1}^{k}\left(\begin{array}{c}
k+i-2 \\
i-1
\end{array}\right)-\sum_{i=1}^{k}\left(\begin{array}{c}
i-2 \\
i-1
\end{array}\right)+1 \\
& =\left(\begin{array}{c}
2 k-1 \\
k-1
\end{array}\right)+1<\left(\begin{array}{c}
2 k \\
k-1
\end{array}\right)-\left(\begin{array}{c}
k \\
k-1
\end{array}\right)-\left(\begin{array}{l}
k-1 \\
k-2
\end{array}\right)+3,
\end{aligned}
$$

which contradicts the assumption that $|\mathcal{H}|>|H M(n, k, 2)|$ and $n>2 k$. Therefore, $a_{k} \geq 2 k$.

Now suppose that $a_{k}=2 k$ and $a_{k-1} \leq 2 k-2$. Then,

$$
\begin{aligned}
|\mathcal{H}| & \leq \sum_{i=h}^{k}\left(\begin{array}{c}
a_{i}-1 \\
i-1
\end{array}\right)-\sum_{i=h}^{k}\left(\begin{array}{c}
a_{i}-1-k \\
i-1
\end{array}\right)+1 \\
& \leq\left(\begin{array}{c}
2 k-1 \\
k-1
\end{array}\right)+\sum_{i=1}^{k-1}\left(\begin{array}{c}
k+i-2 \\
i-1
\end{array}\right)-\sum_{i=1}^{k}\left(\begin{array}{c}
i-2 \\
i-1
\end{array}\right) \\
& =\left(\begin{array}{c}
2 k-1 \\
k-1
\end{array}\right)+\left(\begin{array}{c}
2 k-2 \\
k-2
\end{array}\right)<\left(\begin{array}{c}
2 k \\
k-1
\end{array}\right)-\left(\begin{array}{c}
k \\
k-1
\end{array}\right)-\left(\begin{array}{l}
k-1 \\
k-2
\end{array}\right)+3,
\end{aligned}
$$

where the strict inequality uses $k \geq 4$ and $n>2 k$. This contradicts the assumption that $|\mathcal{H}|>|H M(n, k, 2)|$.

Claim 2.14 implies that $\sum_{i=h}^{k}\left(\begin{array}{c}a_{i}-1-k \\ i-1\end{array}\right)-1>0$. Therefore,

$$
|\mathcal{H}| \leq \sum_{i=h}^{k}\left(\begin{array}{c}
a_{i}-1 \\
i-1
\end{array}\right)-\sum_{i=h}^{k}\left(\begin{array}{c}
a_{i}-1-k \\
i-1
\end{array}\right)+1<\sum_{i=h}^{k}\left(\begin{array}{c}
a_{i}-1 \\
i-1
\end{array}\right),
$$

contradicts the assumption that $|\mathcal{H}|=\sum_{i=h}^{k}\left(\begin{array}{c}a_{i} \\ i\end{array}\right)-\sum_{i=h}^{k}\left(\begin{array}{c}a_{i}-1 \\ i\end{array}\right)=\sum_{i=h}^{k}\left(\begin{array}{c}a_{i}-1 \\ i-1\end{array}\right)$. Therefore, if $\mathcal{H} \subset H M(n, k, 1)$, then $\left|\partial_{\ell} \mathcal{H}\right|>\sum_{i=h}^{k}\left(\begin{array}{c}a_{i} \\ i-\ell\end{array}\right)$, and this completes the proof of Theorem 1.3 .

\subsection{Proof of Theorem 1.6}

In this section we prove Theorem 1.6. We need the following theorem for $t$-intersecting families. 
Theorem 2.15 (Ahlswede and Khachatrian, [2]). Let $t \geq 1, k \geq 3$, and $n>(t+1)(k-$ $t+1)$. Suppose that $\mathcal{H} \subset\left(\begin{array}{c}{[n]} \\ k\end{array}\right)$ is a t-intersecting family and

$$
|\mathcal{H}|=m> \begin{cases}\max \{|A K(n, k, t)|,|E M(n, k, t+2, t+1)|\}, & \text { if } t<\frac{k-1}{2}, \\ |E M(n, k, t+2, t+1)|, & \text { if } t \geq \frac{k-1}{2} .\end{cases}
$$

Then $\mathcal{H} \subset E M(n, k, t, t)$.

Proof of Theorem 1.6. Suppose $\mathcal{H}$ is given as in Theorem 1.6. By Theorem 2.15, $\mathcal{H} \subset$ $\operatorname{EM}(n, k, t, t)$ and by Corollary 2.7, we have $\left|\partial_{\ell} \mathcal{H}\right| \geq\left|\partial_{\ell} L_{m} \operatorname{EM}(n, k, t, t)\right|$.

We now show that the value of $m(n, k, t)$ in the theorem cannot be reduced for $t \geq \frac{k-1}{2}$ and is tight up to a constant multiplicative factor for $t<\frac{k-1}{2}$. Indeed, our construction is $\mathcal{H}=E M(n, k, t+2, t+1)$ and hence it suffices to prove the following.

Fact 2.16. Let $n$ be sufficiently large and $m=|E M(n, k, t+2, t+1)|$. Then

$$
\left|\partial_{\ell} \operatorname{EM}(n, k, t+2, t+1)\right|<\left|\partial_{\ell} L_{m} E M(n, k, t, t)\right| \quad \text { for all } \quad 1 \leq \ell \leq t .
$$

In particular, for $1 \leq \ell \leq t$ the lower bound $m(n, k, t)$ for $|\mathcal{H}|$ in Theorem 1.6 cannot be reduced to be less than $|\operatorname{EM}(n, k, t+2, t+1)| \sim(t+2)\left(\begin{array}{c}n \\ k-t-1\end{array}\right)$.

Note that when $t<\frac{k-1}{2}$ Fact 2.16 implies that the constant multiplicative factor is at most $|A K(n, k, t)| /|E M(n, k, t+2, t+1)| \sim \frac{k-t+1}{t+2}$ which is independent of $n$.

Let $x \in \mathbb{R}$ such that $\left(\begin{array}{c}x-t \\ k-t\end{array}\right)=|E M(n, k, t+2, t+1)|=(t+2)\left(\begin{array}{c}n-t-2 \\ k-t-1\end{array}\right)+\left(\begin{array}{c}n-t-2 \\ k-t-2\end{array}\right)$, then $x=\Theta\left(n^{\frac{k-t-1}{k-t}}\right)$. Applying Lemma 2.4 to $\operatorname{EM}(n, k, t, t)$, we obtain

$$
\begin{aligned}
\left|\partial_{\ell} L_{m} E M(n, k, t, t)\right| & \geq \sum_{i=t-\ell}^{k-\ell}\left(\begin{array}{l}
t \\
i
\end{array}\right)\left(\begin{array}{c}
x-t \\
k-\ell-i
\end{array}\right) \\
& =\left(\begin{array}{c}
t \\
t-\ell
\end{array}\right)\left(\begin{array}{l}
x-t \\
k-t
\end{array}\right)+(1+o(1))\left(\begin{array}{c}
t \\
t-\ell+1
\end{array}\right)\left(\begin{array}{c}
x-t \\
k-t-1
\end{array}\right) \\
& =(t+2)\left(\begin{array}{c}
t \\
t-\ell
\end{array}\right)\left(\begin{array}{c}
n \\
k-t-1
\end{array}\right)+\Theta\left(n^{\frac{(k-t-1)^{2}}{k-t}}\right),
\end{aligned}
$$

and

$$
\begin{aligned}
\left|\partial_{\ell} \operatorname{EM}(n, k, t+2, t+1)\right| & =\sum_{i=t+1-\ell}^{k-\ell}\left(\begin{array}{c}
t+2 \\
i
\end{array}\right)\left(\begin{array}{c}
n-t+2 \\
k-\ell-i
\end{array}\right) \\
& =\left(\begin{array}{c}
t+2 \\
t+1-\ell
\end{array}\right)\left(\begin{array}{c}
n \\
k-t-1
\end{array}\right)+\Theta\left(n^{k-t-2}\right) .
\end{aligned}
$$

If $\ell<t$, then

$$
\left|\partial_{\ell} L_{m} E M(n, k, t, t)\right| \sim(t+2)\left(\begin{array}{c}
t \\
t-\ell
\end{array}\right)\left(\begin{array}{c}
n \\
k-t-1
\end{array}\right)
$$

and

$$
\left|\partial_{\ell} \operatorname{EM}(n, k, t+2, t+1)\right| \sim\left(\begin{array}{c}
t+2 \\
t+1-\ell
\end{array}\right)\left(\begin{array}{c}
n \\
k-t-1
\end{array}\right) .
$$

Since $\left(\begin{array}{c}t+2 \\ t+1-\ell\end{array}\right)<(t+2)\left(\begin{array}{c}t \\ t-\ell\end{array}\right)$ for $\ell<t$,

$$
\left|\partial_{\ell} E M(n, k, t+2, t+1)\right|<\left|\partial_{\ell} L_{m} E M(n, k, t, t)\right|
$$


for large $n$.

If $\ell=t$, then

$$
\left|\partial_{\ell} L_{m} E M(n, k, t, t)\right| \sim(t+2)\left(\begin{array}{c}
n \\
k-t-1
\end{array}\right)+\Theta\left(n^{\frac{(k-t-1)^{2}}{k-t}}\right)
$$

and

$$
\left|\partial_{\ell} \operatorname{EM}(n, k, t+2, t+1)\right| \sim(t+2)\left(\begin{array}{c}
n \\
k-t-1
\end{array}\right)+\Theta\left(n^{k-t-2}\right) .
$$

Since $\frac{(k-t-1)^{2}}{k-t}>k-t-2$,

$$
\left|\partial_{t+1} E M(n, k, t+2, t+1)\right|<\left|\partial_{t+1} L_{m} E M(n, k, t, t)\right|
$$

for large $n$. Consequently, Fact 2.16 holds and the proof is complete.

\subsection{Proof of Theorem 1.10}

Before proving Theorem 1.10 we need some structure theorems for a family with large size and a given matching number.

Definition 2.17. Let $n \geq s k+1, k \geq 3$, and $s \geq 1$. Let $v_{0}, \ldots, v_{s-1} \in[n]$ be distinct vertices, $T_{1}, \ldots, T_{s} \subset[n]$ be pairwise disjoint $k$-sets, and $v_{i} \in T_{i}$ for all $1 \leq i \leq s-1$, and $v_{0} \notin T_{i}$ for all $1 \leq i \leq s$. Let

$$
\begin{aligned}
P F(n, k, s)= & \left\{T_{1}, \ldots, T_{s}\right\} \cup \\
& \left\{A \in\left(\begin{array}{c}
{[n]} \\
k
\end{array}\right): \exists 0 \leq i \leq s-1 \text { such that } x_{i} \in A \text { and }\left|A \cap \bigcup_{j=i+1}^{s} T_{i}\right| \geq 1\right\} .
\end{aligned}
$$

Notice that $|P F(n, k, s)| \sim k\left(\begin{array}{c}s+1 \\ 2\end{array}\right)\left(\begin{array}{c}n \\ k-2\end{array}\right)$.

Theorem 2.18 (Kostochka and Mubayi, 20]). For every $k \geq 3, s \geq t \geq 2$, there exists

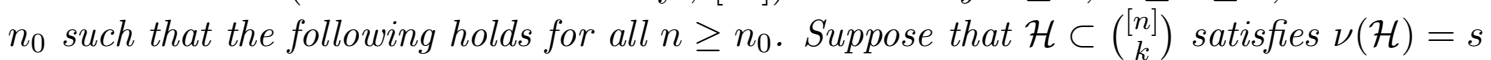
and

$$
|\mathcal{H}|> \begin{cases}|E M(n, 3, s-t, 1)|+|E M(n-s+t, 3,2 s+1,2)| & \text { if } k=3, \\ |E M(n, k, s-t, 1)|+|P F(n-s+t, k, t)| & \text { if } k \geq 4 .\end{cases}
$$

Then there exists $X \subset[n]$ with $|X|=s-t+1$ such that $\nu(H-X)=t-1$. The bound on $|\mathcal{H}|$ is tight. In particular, if

$$
|\mathcal{H}|> \begin{cases}|E M(n, 3,2 s+1,2)| & \text { for } k=3, \\ |P F(n, k, s)| & \text { for } k \geq 4,\end{cases}
$$

then there exists $v \in[n]$ such that $\nu(H-v)=s-1$.

Note that the proof of Theorem 2.18 was not included in [20], but one can easily prove it using results in [11] (Theorems 4.1 and 4.2 in [11]).

We also need the following structure theorems for intersecting families. Let

$$
\text { - } H_{0}^{3}(n)=\left\{A \in\left(\begin{array}{c}
{[n]} \\
3
\end{array}\right):|A \cap[3]| \geq 2\right\} \text {. }
$$


- $H_{1}^{3}(n)=\left\{A \in\left(\begin{array}{c}{[n]} \\ 3\end{array}\right): 1 \in A\right.$ and $\left.|A \cap\{2,3,4\}| \geq 1\right\} \cup\{234\}$.

- $H_{2}^{3}(n)=\left\{A \in\left(\begin{array}{c}{[n]} \\ 3\end{array}\right): 1 \in A\right.$ and $\left.|A \cap\{2,3\}| \geq 1\right\} \cup\{234,235,145\}$.

- $H_{3}^{3}(n)=\left\{A \in\left(\begin{array}{c}{[n]} \\ 3\end{array}\right):\{1,2\} \in A\right\} \cup\{134,135,145,234,235,245\}$.

- $H_{4}^{3}(n)=\left\{A \in\left(\begin{array}{c}{[n]} \\ 3\end{array}\right):\{1,2\} \in A\right\} \cup\{134,156,235,236,245,246\}$.

- $H_{5}^{3}(n)=\left\{A \in\left(\begin{array}{c}{[n]} \\ 3\end{array}\right):\{1,2\} \in A\right\} \cup\{134,156,136,235,236,246\}$.

- For $k \geq 4$ and $0 \leq i \leq 5, H_{i}^{k}(n)=\left\{A \in\left(\begin{array}{c}{[n]} \\ k\end{array}\right): \exists B \in H_{i}^{3}(n)\right.$ such that $\left.B \subset A\right\}$.

Fact 2.19. The following holds for all $n \geq k \geq 3$.

- $\left|H_{0}^{k}(n)\right|=3\left(\begin{array}{c}n-3 \\ k-2\end{array}\right)+\left(\begin{array}{c}n-3 \\ k-3\end{array}\right)<3\left(\begin{array}{c}n \\ k-2\end{array}\right)-2\left(\begin{array}{c}n \\ k-3\end{array}\right)$.

- $\left|H_{1}^{k}(n)\right|=3\left(\begin{array}{c}n-4 \\ k-2\end{array}\right)+4\left(\begin{array}{c}n-4 \\ k-3\end{array}\right)+\left(\begin{array}{c}n-4 \\ k-4\end{array}\right)<3\left(\begin{array}{c}n \\ k-2\end{array}\right)-2\left(\begin{array}{c}n \\ k-3\end{array}\right)$.

- $\max \left\{\left|H_{i}^{k}(n)\right|: 2 \leq i \leq 5\right\} \leq 2\left(\begin{array}{c}n \\ k-2\end{array}\right)$.

Definition 2.20. Let $n \geq 2 k$ and $k \geq 3$. Let $Y=[2, k+1], Z=[k+2,2 k]$. The n-vertex $k$-graph $P F(n, k)$ consists of all $k$-subsets of $[n]$ containing a member of the family

$$
\begin{aligned}
G= & \{A: 1 \in A \text { and }|A \cap Y|=1 \text { and }|A \cap Z|=1\} \cup \\
& \{Y,\{1, k, k+1\}, Z \cup\{k\}, Z \cup\{k+1\}\} .
\end{aligned}
$$

Note that $|P F(n, k)|=O\left(n^{k-3}\right)$.

Theorem 2.21 (Kostochka and Mubayi, [20]). Let $k \geq 4$ be fixed and $n$ be sufficiently large. Then there is $C>0$ such that for every intersecting $n$-vertex $k$-graph $\mathcal{H}$ with $|\mathcal{H}|>|P F(n, k)|=O\left(n^{k-3}\right)$, one can remove from $\mathcal{H}$ at most $C n^{k-4}$ edges so that the resulting $k$-graph $\mathcal{H}^{\prime}$ is contained in one of $H_{0}^{k}(n), \ldots, H_{5}^{k}(n), E K R(n, k)$.

For intersecting 3-graphs there is a stronger result. Define

$$
\tau(\mathcal{H})=\min \{|S|: S \subset V(\mathcal{H}) \text { and }|S \cap A| \geq 1 \text { for all } A \in \mathcal{H}\} .
$$

Theorem 2.22 (Kostochka and Mubayi, [20]). Let $\mathcal{H}$ be an intersecting 3-graph and $n=|V(\mathcal{H})| \geq 6$. If $\tau(\mathcal{H}) \leq 2$, then $\mathcal{H}$ is contained in one of $E K R(n, 3), H_{1}^{3}(n), \ldots, H_{5}^{3}(n)$.

The following result shows that the size of an intersecting 3 -graph $\mathcal{H}$ with $\tau(\mathcal{H}) \geq 3$ is bounded by a constant.

Theorem 2.23 (Frankl, [7]). Let $k \geq 3$ and $n$ be sufficiently large. Then every intersecting $n$-vertex $k$-graph $\mathcal{H}$ with $\tau(\mathcal{H}) \geq 3$ satisfies $|\mathcal{H}| \leq|P F(n, k)|$. Moreover, if $k \geq 4$, then equality holds only if $\mathcal{H} \cong P F(n, k)$.

Now we are ready to prove Theorem 1.10 . 
Proof of Theorem 1.10. Let $n$ be sufficiently large and $c=c(k, s)$ be given by (4). We may assume that $\mathcal{H}$ is shifted and $\nu(\mathcal{H})=s$. For every $v \in[n]$ let $d_{\mathcal{H}}(v)=|\{A \in \mathcal{H}: v \in A\}|$, and let $\Delta=\max \left\{d_{\mathcal{H}}(v): v \in[n]\right\}$. Suppose that $m=\sum_{i=h}^{k}\left(\begin{array}{c}a_{i} \\ i\end{array}\right)-\sum_{i=h}^{k}\left(\begin{array}{c}a_{i}-s \\ i\end{array}\right)$ for some integers $a_{k}>\cdots>a_{h} \geq h \geq 1$. Then by Lemma 2.4, it suffice to show that

$$
|\partial \mathcal{H}| \geq \sum_{i=h}^{k}\left(\begin{array}{c}
a_{i} \\
i-1
\end{array}\right)=\left|\partial L_{m} E M(n, k, s, 1)\right| .
$$

Note that $a_{k} \rightarrow \infty$ as $n \rightarrow \infty$ and so $m \sim s\left(\begin{array}{c}a_{k}-1 \\ k-1\end{array}\right)$.

Claim 2.24. $\Delta \leq \sum_{i=h}^{k}\left(\begin{array}{c}a_{i}-1 \\ i-1\end{array}\right)=(1+o(1)) \frac{m}{s}$.

Proof of Claim 2.24. Suppose that there exists $v \in[n]$ with $d_{\mathcal{H}}(v)>\sum_{i=h}^{k}\left(\begin{array}{c}a_{i}-1 \\ i-1\end{array}\right)$. Let $\mathcal{H}(v)=\{A \backslash\{v\}: v \in A \in \mathcal{H}\}$. Then by the Kruskal-Katona theorem,

$$
|\partial \mathcal{H}| \geq|\mathcal{H}(v)|+|\partial \mathcal{H}(v)|>\sum_{i=h}^{k}\left(\begin{array}{c}
a_{i}-1 \\
i-1
\end{array}\right)+\sum_{i=h}^{k}\left(\begin{array}{c}
a_{i}-1 \\
i-2
\end{array}\right)=\sum_{i=h}^{k}\left(\begin{array}{c}
a_{i} \\
i-1
\end{array}\right),
$$

and we are done.

We are going to use Theorem 2.18 and Claim 2.24 to define a sequence of distinct vertices $v_{1}, \ldots, v_{s-1}$ and a sequence of $k$-graphs $\mathcal{H}_{1}, \ldots, \mathcal{H}_{s-1}$ such that $\nu\left(\mathcal{H}_{i}\right)=s-i$ and $\left|\mathcal{H}_{i}\right|>$ $(1-o(1)) \frac{s-i}{s} m$ for all $1 \leq i \leq s-1$. Since $\mathcal{H}$ is shifted, we may assume that $v_{i}=i$ for $1 \leq i \leq s-1$.

First, by the assumption on the size of $\mathcal{H}$ and Theorem [2.18, there exists $v_{1} \in[n]$ such that $\mathcal{H}_{1}:=\mathcal{H}-v_{1}$ satisfies $\nu\left(\mathcal{H}_{1}\right)=s-1$. By Claim 2.24, $d_{\mathcal{H}}\left(v_{1}\right)<(1+o(1)) m / s$, so $\left|\mathcal{H}_{1}\right|>(1-o(1)) \frac{s-1}{s} m$.

Now suppose that we have defined $\mathcal{H}_{i}$ for some $1 \leq i \leq s-2$ such that $\nu\left(\mathcal{H}_{i}\right)=s-i$ and $\left|\mathcal{H}_{i}\right|>(1-o(1)) \frac{s-i}{s} m$. Since

$$
\left|\mathcal{H}_{i}\right|>(1-o(1)) \frac{s-i}{s} m \geq \frac{s-i}{s} c\left(\begin{array}{c}
n \\
k-2
\end{array}\right) \geq \begin{cases}|E M(n, 3,2(s-i)+1,2)|, & \text { for } k=3, \\
|P F(n, k, s-i)|, & \text { for } k \geq 4,\end{cases}
$$

by Theorem [2.18, there exists $v_{i+1} \in[n]$ such that $\mathcal{H}_{i+1}:=\mathcal{H}_{i}-v_{i+1}$ satisfies $\nu\left(\mathcal{H}_{i+1}\right)=$ $s-i-1$. By Claim 2.24, $\left|\mathcal{H}_{i+1}\right|>(1-o(1)) \frac{s-i-1}{s} m$.

Note that $\mathcal{H}_{s-1}$ satisfies $\nu\left(\mathcal{H}_{s-1}\right)=1$ and

$$
\left|\mathcal{H}_{s-1}\right|>(1-o(1)) \frac{1}{s} m \geq \frac{1}{s} c\left(\begin{array}{c}
n \\
k-2
\end{array}\right) \geq 3\left(\begin{array}{c}
n \\
k-2
\end{array}\right)>|P F(n, k)| .
$$

If $k=3$, then by Theorem 2.23, $\tau\left(\mathcal{H}_{s-1}\right) \leq 2$. Therefore, by Theorem 2.22, $\mathcal{H}$ is contained in one of $\operatorname{EKR}(n, 3), H_{1}^{3}(n), \ldots, H_{5}^{3}(n)$. Since $\left|\mathcal{H}_{s-1}\right|>3 n$ and by Fact 2.19, $\max _{0 \leq i \leq 5}\left\{\left|H_{i}^{3}\right|\right\} \leq 3 n-8$, we must have $\mathcal{H} \subset E K R(n, 3)=E M(n, 3,1,1)$. Note that $\mathcal{H}_{s-1}$ is obtained from $\mathcal{H}$ by removing $s-1$ vertices, so $\mathcal{H} \subset E M(n, 3, s, 1)$. Therefore, by Lemma 2.5. $|\partial \mathcal{H}| \geq\left|\partial L_{m} E M(n, 3, s, 1)\right|$ and we are done.

Now we may assume that $k \geq 4$. Then, by Theorem 2.21, one can remove at most $C n^{k-4}$ edges from $\mathcal{H}_{s-1}$ such that the resulting $k$-graph $\mathcal{H}^{\prime}$ is contained in one of $H_{0}^{k}(n), \ldots, H_{5}^{k}(n)$, $\operatorname{EKR}(n, k)$. Note that $\left|\mathcal{H}^{\prime}\right| \geq\left|\mathcal{H}_{s-1}\right|-C n^{k-4}>3\left(\begin{array}{c}n \\ k-2\end{array}\right)-\left(\begin{array}{c}n \\ k-3\end{array}\right)$ and by Fact 2.19, 
$\max _{0 \leq i \leq 5}\left\{\left|H_{i}^{k}\right|\right\}<3\left(\begin{array}{c}n \\ k-2\end{array}\right)-2\left(\begin{array}{c}n \\ k-3\end{array}\right)$, so $\mathcal{H}^{\prime} \subset \operatorname{EKR}(n, k)=\operatorname{EM}(n, k, 1,1)$. Here we need $n$ to be sufficient large so that $C n^{k-4}<\left(\begin{array}{c}n \\ k-3\end{array}\right)$.

Note that $\mathcal{H}_{s-1}$ is obtained from $\mathcal{H}$ by removing $s-1$ vertices. If $\mathcal{H}_{s-1} \subset E M(n, k, 1,1)$, then $\mathcal{H} \subset E M(n, k, s, 1)$ and by Lemma 2.5 we are done. So we may assume that $\mathcal{H}_{s-1} \not \subset$ $\operatorname{EM}(n, k, 1,1)$, i.e. $\mathcal{H}_{s-1} \backslash \mathcal{H}^{\prime} \neq \emptyset$. Let $A \in \mathcal{H}_{s-1} \backslash \mathcal{H}^{\prime}$ and since $\mathcal{H}_{s-1}$ is shifted, we may assume that $A=\{s+1, \ldots, s+k\}$. Since $\mathcal{H}_{s-1}$ is intersecting, every edge in $\mathcal{H}^{\prime}$ must have nonempty intersecting with $A$. So $\mathcal{H}^{\prime} \subset H M(n, k, 1, k)$. This implies that one can remove at most $C n^{k-4}$ edges from $\mathcal{H}$ such that the resulting $k$-graph $\mathcal{H}^{\prime \prime}$ satisfies $\mathcal{H}^{\prime \prime} \subset H M(n, k, s, k)$.

Let $y \in \mathbb{R}$ satisfy $\left|\mathcal{H}^{\prime \prime}\right|=\left(\begin{array}{c}y \\ k\end{array}\right)-\left(\begin{array}{c}y-s \\ k\end{array}\right)-\left(\begin{array}{c}y-s-k \\ k-1\end{array}\right)$. Then by Lemma 2.12, $|\partial \mathcal{H}| \geq\left|\partial \mathcal{H}^{\prime \prime}\right| \geq$ $\left(\begin{array}{c}y \\ k-1\end{array}\right)$. Let $x \in \mathbb{R}, a_{k}, \ldots, a_{h} \in \mathbb{N}$ such that $a_{k}>\cdots>a_{h} \geq h \geq 1$ and $|\mathcal{H}|=\left(\begin{array}{c}x \\ k\end{array}\right)-\left(\begin{array}{c}x-s \\ k\end{array}\right)=$ $\sum_{i=h}^{k}\left(\begin{array}{c}a_{i} \\ i\end{array}\right)-\sum_{i=h}^{k}\left(\begin{array}{c}a_{i}-s \\ i\end{array}\right)$. It is easy to see that $x \leq a_{k}+1$.

Claim 2.25. $y>x+1$.

Proof of Claim 2.25. Suppose not. Then

$$
\begin{aligned}
& \left(\begin{array}{c}
x+1 \\
k
\end{array}\right)-\left(\begin{array}{c}
x+1-s \\
k
\end{array}\right)-\left(\begin{array}{c}
x+1-s-k \\
k-1
\end{array}\right) \\
\geq & \left(\begin{array}{l}
y \\
k
\end{array}\right)-\left(\begin{array}{c}
y-s \\
k
\end{array}\right)-\left(\begin{array}{c}
y-s-k \\
k-1
\end{array}\right) \geq\left(\begin{array}{l}
x \\
k
\end{array}\right)-\left(\begin{array}{c}
x-s \\
k
\end{array}\right)-C n^{k-4} .
\end{aligned}
$$

Since $|\mathcal{H}| \geq c\left(\begin{array}{c}n \\ k-2\end{array}\right), x=\Omega\left(n^{\frac{k-2}{k-1}}\right)$. Therefore,

$$
\begin{aligned}
& \left(\begin{array}{c}
x+1 \\
k
\end{array}\right)-\left(\begin{array}{c}
x+1-s \\
k
\end{array}\right)-\left(\begin{array}{c}
x+1-s-k \\
k-1
\end{array}\right) \\
= & \left(\begin{array}{l}
x \\
k
\end{array}\right)-\left(\begin{array}{c}
x-s \\
k
\end{array}\right)+\left(\begin{array}{c}
x \\
k-1
\end{array}\right)-\left(\begin{array}{c}
x-s \\
k-1
\end{array}\right)-\left(\begin{array}{c}
x+1-s-k \\
k-1
\end{array}\right) \\
< & \left(\begin{array}{l}
x \\
k
\end{array}\right)-\left(\begin{array}{c}
x-s \\
k
\end{array}\right)-\frac{1}{2}\left(\begin{array}{c}
x-s-k \\
k-1
\end{array}\right) \\
< & \left(\begin{array}{l}
x \\
k
\end{array}\right)-\left(\begin{array}{c}
x-s \\
k
\end{array}\right)-C n^{k-4},
\end{aligned}
$$

a contradiction. This completes the proof of Claim 2.25,

By Claim 2.25,

$$
|\partial \mathcal{H}| \geq\left(\begin{array}{c}
y \\
k-1
\end{array}\right)>\left(\begin{array}{l}
x+1 \\
k-1
\end{array}\right) \geq \sum_{i=h}^{k}\left(\begin{array}{c}
a_{i} \\
i-1
\end{array}\right) .
$$

This completes the proof of Theorem 1.10 .

\section{Concluding Remarks}

Let $\mathcal{H} \subset\left(\begin{array}{c}{[n]} \\ 3\end{array}\right)$ be an intersecting family with $|\mathcal{H}| \geq P F(n, 3)=10$. Then Theorems 2.22 and 2.23 completely determine the structure of $\mathcal{H}$. One can use this structural result to determine the minimum size of $\left|\partial_{\ell} \mathcal{H}\right|$ completely for $1 \leq \ell \leq 2$. However, the calculation is very complicated and tedious, so we omit it here. 
As we mentioned before, for $1 \leq \ell \leq t$ the lower bound for $|\mathcal{H}|$ in Theorem [1.6 above is tight for $t \geq \frac{k-1}{2}$ and can be improved for $t<\frac{k-1}{2}$. Indeed, one can use the $\Delta$-system method (see [20]) to prove the following result.

Theorem 3.1. Let $t \geq 1, k \geq 3, \epsilon>0$, and $n$ be sufficiently large. Suppose that $\mathcal{H} \subset\left(\begin{array}{c}{[n]} \\ k\end{array}\right)$ is t-intersecting. If $t<\frac{k-1}{2}$ and $|\mathcal{H}|>(k-t+\epsilon)\left(\begin{array}{c}n \\ k-t-1\end{array}\right)$, then either $\mathcal{H} \subset A K(n, k, t)$ or $\mathcal{H} \subset \operatorname{EM}(n, k, t, t)$. If $t \geq \frac{k-1}{2}$ and $|\mathcal{H}|>(t+1+\epsilon)\left(\begin{array}{c}n \\ k-t-1\end{array}\right)$, then $\mathcal{H}$ is contained in one of $A K(n, k, t), E M(n, k, t+2, t+1), E M(n, k, t, t)$.

One can easily use Corollary 2.7 to show that for $1 \leq \ell \leq t,\left|\partial_{\ell} L_{m} A K(n, k, t)\right|>$ $\left|\partial_{\ell} L_{m} \operatorname{EM}(n, k, t, t)\right|$ for sufficiently large $n$ and $m$. Therefore, by Theorem 3.1, we obtain the following result.

Fact 3.2. Let $k \geq 3, t<\frac{k-1}{2}, 1 \leq \ell \leq t, \epsilon>0$, and $n$ be sufficiently large. Then every $t$-intersecting family $\mathcal{H} \subset\left(\begin{array}{c}{[n]} \\ k\end{array}\right)$ with $|\mathcal{H}|=m>(k-t+\epsilon)\left(\begin{array}{c}n \\ k-t-1\end{array}\right)$ satisfies $\left|\partial_{\ell} \mathcal{H}\right| \geq$ $\left|\partial_{\ell} L_{m} \operatorname{EM}(n, k, t, t)\right|$.

\section{References}

[1] R. Ahlswede, H. Aydinian, and L. H. Khachatrian. On shadows of intersecting families. Combinatorica, 24(4):555-566, 2004.

[2] R. Ahlswede and L. H. Khachatrian. The complete nontrivial-intersection theorem for systems of finite sets. J. Combin. Theory Ser. A, 76(1):121-138, 1996.

[3] P. Borg and C. Feghali. On the hilton-spencer intersection theorems for unions of cycles. arXiv preprint arXiv:1908.08825, 2019.

[4] G. F. Clements and H.-D. O. F. Gronau. On maximal antichains containing no set and its complement. Discrete Math., 33(3):239-247, 1981.

[5] P. Erdős. A problem on independent r-tuples. Ann. Univ. Sci. Budapest. Eötvös Sect. Math., 8:93-95, 1965.

[6] P. Erdős, C. Ko, and R. Rado. Intersection theorems for systems of finite sets. Quart. J. Math. Oxford Ser. (2), 12:313-320, 1961.

[7] P. Frankl. On intersecting families of finite sets. Bull. Austral. Math. Soc., 21(3):363$372,1980$.

[8] P. Frankl. The shifting technique in extremal set theory. In Surveys in combinatorics 1987 (New Cross, 1987), volume 123 of London Math. Soc. Lecture Note Ser., pages 81-110. Cambridge Univ. Press, Cambridge, 1987.

[9] P. Frankl. Shadows and shifting. Graphs Combin., 7(1):23-29, 1991.

[10] P. Frankl. Improved bounds for Erdős' matching conjecture. J. Combin. Theory Ser. A, 120(5):1068-1072, 2013.

[11] P. Frankl. Resilient hypergraphs with fixed matching number. Combinatorica, 38(5):1079-1094, 2018.

[12] P. Frankl and Z. Füredi. On hypergraphs without two edges intersecting in a given number of vertices. J. Combin. Theory Ser. A, 36(2):230-236, 1984. 
[13] P. Frankl and Z. Füredi. Extremal problems concerning Kneser graphs. J. Combin. Theory Ser. B, 40(3):270-284, 1986.

[14] P. Frankl and Z. Füredi. A new short proof of the EKR theorem. J. Combin. Theory Ser. A, 119(6):1388-1390, 2012.

[15] P. Frankl and N. Tokushige. Some best possible inequalities concerning crossintersecting families. J. Combin. Theory Ser. A, 61(1):87-97, 1992.

[16] H.-D. O. F. Gronau. On Sperner families in which no $k$ sets have an empty intersection. J. Combin. Theory Ser. A, 28(1):54-63, 1980.

[17] J. Han and Y. Kohayakawa. The maximum size of a non-trivial intersecting uniform family that is not a subfamily of the Hilton-Milner family. Proc. Amer. Math. Soc., 145(1):73-87, 2017.

[18] G. Katona. Intersection theorems for systems of finite sets. Acta Math. Acad. Sci. Hungar., 15:329-337, 1964.

[19] G. Katona. A theorem of finite sets. In Theory of graphs (Proc. Colloq., Tihany, 1966), pages 187-207, 1968.

[20] A. Kostochka and D. Mubayi. The structure of large intersecting families. Proc. Amer. Math. Soc., 145(6):2311-2321, 2017.

[21] J. B. Kruskal. The number of simplices in a complex. In Mathematical optimization techniques, pages 251-278. Univ. of California Press, Berkeley, Calif., 1963.

[22] A. Mammoliti and T. Britz. On Mubayi's conjecture and conditionally intersecting sets. SIAM J. Discrete Math., 32(3):2361-2380, 2018.

[23] A. Sali. Some intersection theorems. Combinatorica, 12(3):351-361, 1992.

[24] X. Y. Zha. On a conjecture on the Sperner property. European J. Combin., 10(6):603607, 1989. 